\title{
Frequency measurements of the gyrotrons used for collective Thomson scattering diagnostics at TEXTOR and ASDEX Upgrade
}

P. Woskov, H. Bindslev, F. Leipold, F. Meo, S. K. Nielsen, E. L. Tsakadze, S. B. Korsholm, J. Scholten, C. Tito, E. Westerhof, J. W. Oosterbeek, F. Leuterer, F. Monaco, M. Muenich, and D. Wagner

Citation: Review of Scientific Instruments 77, 10E524 (2006);

View online: https://doi.org/10.1063/1.2347694

View Table of Contents: http://aip.scitation.org/toc/rsi/77/10

Published by the American Institute of Physics

\section{Articles you may be interested in}

Self-consistent non-stationary theory of the gyrotron

Physics of Plasmas 23, 083125 (2016); 10.1063/1.4961962

Startup and mode competition in a $420 \mathrm{GHz}$ gyrotron

Physics of Plasmas 24, 093102 (2017); 10.1063/1.4997910

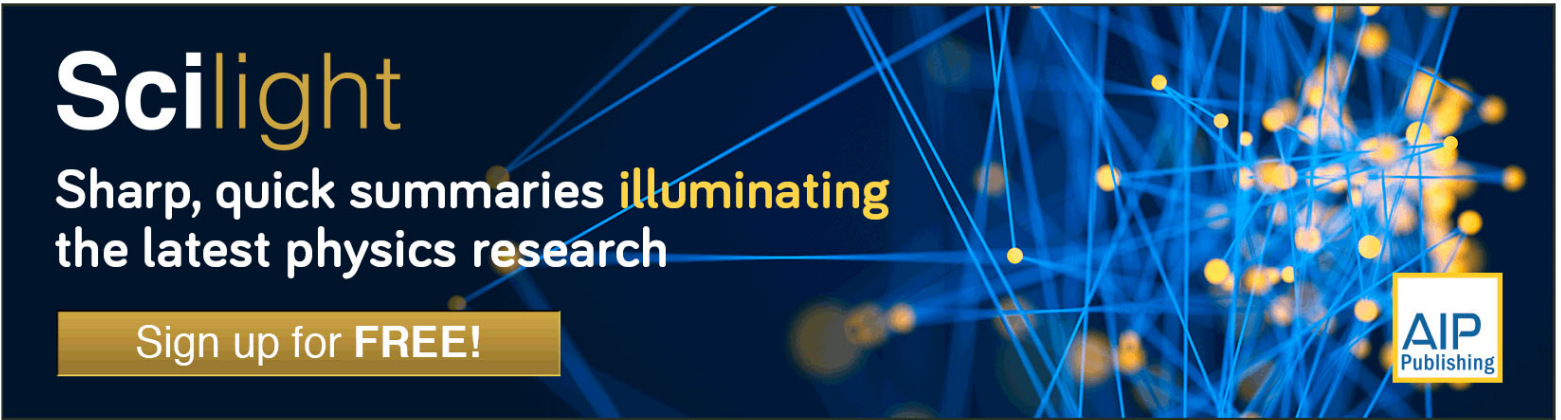




\title{
Frequency measurements of the gyrotrons used for collective Thomson scattering diagnostics at TEXTOR and ASDEX Upgrade
}

\author{
P. Woskov \\ MIT Plasma Science and Fusion Center, Cambridge, Massachusetts 02139 \\ H. Bindslev, F. Leipold, F. Meo, S. K. Nielsen, and E. L. Tsakadze \\ Ris $\varnothing$ National Laboratory, Association EURATOM, DK-4000 Roskilde, Denmark \\ S. B. Korsholm \\ MIT Plasma Science and Fusion Center, Cambridge, Massachusetts 02139 \\ and Ris $\phi$ National Laboratory, Association EURATOM, DK-4000 Roskilde, Denmark \\ J. Scholten, C. Tito, and E. Westerhof \\ FOM-Institute for Plasma Physics Rijnhuizen, Association EURATOM-FOM, Trilateral Euregio Cluster, \\ NL-3430 BE Nieuwegein, The Netherlands \\ J. W. Oosterbeek \\ Forschungszentrum Jülich GmbH, Institut für Plasmaphysik, Association EURATOM, D-52428 Jülich, \\ Germany \\ F. Leuterer, F. Monaco, M. Muenich, and D. Wagner \\ Max-Planck-Institut für Plasmaphysik, EURATOM Association, D-85748 Garching, Germany
}

(Received 4 May 2006; presented on 8 May 2006; accepted 3 August 2006;

published online 19 October 2006)

\begin{abstract}
High resolution frequency measurements of the $110 \mathrm{GHz}$ gyrotron at TEXTOR and the $105 \mathrm{GHz}$ mode of the two-frequency gyrotron (Odissey-1) at ASDEX Upgrade (AUG) have been made in support of fast ion collective Thomson scattering diagnostics. Measurements were done by harmonic heterodyne methods using both fast Fourier transform spectroscopy with digital oscilloscopes and fast scanning spectrum analyzers. Accurate frequencies were obtained with a frequency counter. At TEXTOR, at $180 \mathrm{~kW}$ forward power the starting frequency was 109.970 $\pm 0.005 \mathrm{GHz}$ and chirped down as much as $27 \mathrm{MHz}$ depending on the duty factor. At AUG, at $500 \mathrm{~kW}$ forward power the frequency started at $104.786 \mathrm{GHz}$ and chirped down $104 \mathrm{MHz}$, with $90 \%$ of the chirp occurring in the first $100 \mathrm{~ms}$. Plasma perturbation of the TEXTOR gyrotron was observed when both ion cyclotron resonance heating antennas and neutral beam injection were operating, producing modulation at 29 and $58 \mathrm{MHz}$ in the gyrotron output. Each gyrotron was observed to have an instrumental measurement limited linewidth of $120 \mathrm{kHz}$ full width at half maximum. () 2006 American Institute of Physics. [DOI: 10.1063/1.2347694]
\end{abstract}

\section{INTRODUCTION}

The application of gyrotron radiation to fast ion collective Thomson scattering (CTS) diagnostics ${ }^{1,2}$ requires precise knowledge and control of the gyrotron frequency and spectrum. The gyrotron frequency must be accurately known and reproducible to tune narrow receiver notch filters to reject stray background signal, and the gyrotron linewidth including spurious modes must be less than $100 \mathrm{MHz}$ over a large dynamic range $(>100 \mathrm{~dB})$ out to bandwidths of $\pm 6 \mathrm{GHz}$ around the center frequency to provide enough resolution and bandwidth for fast ion measurements. Even more precise knowledge of the frequency to $<1 \mathrm{MHz}$ is required for plasma rotation measurements.

Many factors determine the gyrotron frequency and spectrum. The gyrotron frequency and spurious mode content are affected by the dimensions of the resonator cavity and through the control of the operating parameters including the magnetic field, beam voltage, and current. ${ }^{3}$ Plasma backreflection $^{4}$ and external interference in the environment of a major tokamak experimental facility from other high power electrical systems can also perturb the gyrotron. Previous gyrotron frequency measurements have been limited to laboratory environments and to low average power ${ }^{3,5}$ or low power high cavity $q$ devices, ${ }^{6}$ or to peak frequency measurements. $^{7}$ This article summarizes precise, high resolution spectrum measurements of the high power $110 \mathrm{GHz}$ gyrotron at TEXTOR (Ref. 8) and the $105 \mathrm{GHz}$ mode of the two-frequency gyrotron (Odissey-1) at ASDEX upgrade ${ }^{9}$ (AUG) being used for fast ion CTS diagnostics. The TEXTOR measurements were made during plasma diagnostic operation and resulted in the first observation of a gyrotron spectrum being perturbed by auxiliary heating in a tokamak.

\section{EXPERIMENTAL SETUP}

The TEXTOR and AUG gyrotron spectra were each monitored by two heterodyne systems, as shown in Fig. 1, sharing the signal from a single pickoff horn near the gyrotron output. One heterodyne system was a commercial spec- 


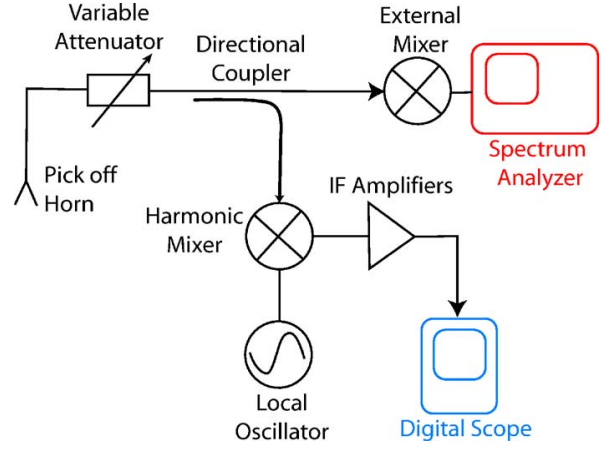

FIG. 1. Experimental setup for gyrotron frequency measurements.

trum analyzer with an external mixer, which was an Agilent E4407B at TEXTOR and a Tektronix 492P at AUG. These spectrum analyzers were used to take repetitive frequency sweeps on about a $2 \mathrm{~ms}$ time scale over typically a $200 \mathrm{MHz}$ bandwidth with a resolution bandwidth of $1 \mathrm{MHz}$. The second heterodyne system used a Pacific Millimeter Products MOD WM harmonic mixer operated at the tenth harmonic with an OMIYIG model YOM2228D yttrium iron garnet (YIG) tuned Gunn local oscillator (LO) to downshift the gyrotron spectrum for fast Fourier transform (FFT) spectroscopy by a digital oscilloscope. At TEXTOR the scope was a Tektronix TDS3032B and at AUG it was a LeCroy LC334AM. The digital scopes were operated at a $1 \mathrm{Gsample/s} \mathrm{sampling} \mathrm{rate} \mathrm{to} \mathrm{obtain} \mathrm{one} 10000$ point data set per gyrotron trigger, which resulted in a $10 \mu$ s long measurement sample with a maximum bandwidth of $500 \mathrm{MHz}$ and a resolution bandwidth of about $120 \mathrm{kHz}$. The gyrotron frequency as a function of time during a pulse was studied on a shot to shot basis, by varying the trigger delay. Accurate frequency measurements were obtained by using the frequency counter option of the Agilent spectrum analyzer at TEXTOR or an EIP model 548 frequency counter at AUG to measure LO frequency of the harmonic mixer.

\section{MEASUREMENTS AT TEXTOR}

The frequency of the TEXTOR $110 \mathrm{GHz}$ gyrotron chirped downward every pulse by a rate and a magnitude dependent on the average power output of the gyrotron, as would be expected by thermal expansion of the resonant cavity. Figure 2 shows this behavior for a peak output power of about $180 \mathrm{~kW}$ for three different duty factors. The starting

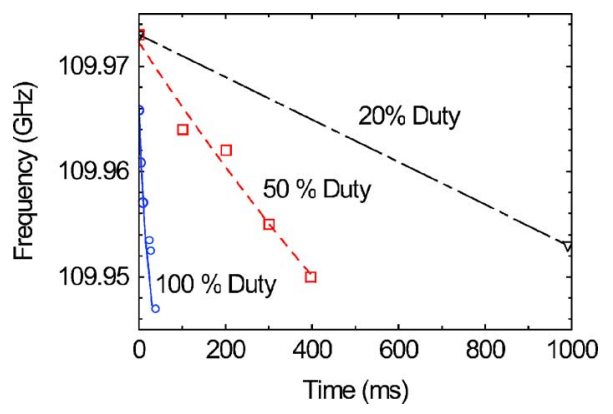

FIG. 2. TEXTOR $110 \mathrm{GHz}$ gyrotron frequency dependence on pulse length and duty factor for approximately $180 \mathrm{~kW}$ output power. The gyrotron frequency was $109.960 \mathrm{GHz}$ as measured with the spectrum analyzer.

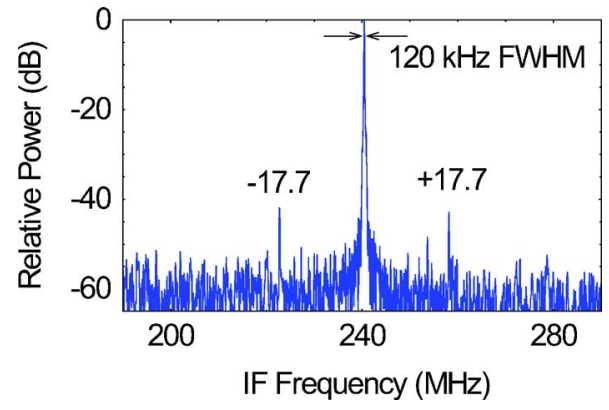

FIG. 3. TEXTOR $110 \mathrm{GHz}$ gyrotron FFT spectrum downshifted to an intermediate frequency showing spurious $\pm 17.7 \mathrm{MHz}$ components that are sometimes present.

frequency at this peak power setting occurs at $109.970 \pm 0.005 \mathrm{GHz}$ as determined by many measurements over different days. The downshift was $27 \mathrm{MHz}$ for a continuous $40 \mathrm{~ms}$ pulse, $23 \mathrm{MHz}$ for a $50 \%$ duty factor pulse with $2 \mathrm{~ms}$ on and $2 \mathrm{~ms}$ off for $400 \mathrm{~ms}$, and $20 \mathrm{MHz}$ for a $20 \%$ duty factor pulse with $2 \mathrm{~ms}$ on and $8 \mathrm{~ms}$ off for $1 \mathrm{~s}$. Typical operation for fast ion CTS diagnostics requires on/ off modulation to be able to subtract the background noise from the weak scattered signal.

The frequency spectrum of the gyrotron was found to be generally clean during most tokamak operations over the observed bandwidth and a dynamic range of up to $50 \mathrm{~dB}$, except for weak $17.5 \pm 0.3 \mathrm{MHz}$ satellites $<-30 \mathrm{~dB}$ relative to the main peak, as shown in Fig. 3, and during combined ion cyclotron resonance heating (ICRH) and neutral beam injection (NBI) heating when ICRH $(29 \mathrm{MHz})$ and harmonic satellites $(58 \mathrm{MHz})$ were present in the gyrotron spectrum under some conditions. The $17.5 \mathrm{MHz}$ satellites are not always present in the gyrotron spectrum and can appear whether TEXTOR is operating or not. Their origin is not yet understood.

The effect of combined ICRH and NBI heating on the gyrotron spectrum is shown in the top graph of Fig. 4 for plasma shot 100472. This spectrum is representative of several plasma shots under similar conditions. The gyrotron beam launch angle was $-5^{\circ}$, that is $5^{\circ}$ to the left from the low field side (LFS) midplane as viewed from the LFS to the high field side (HFS) (see Ref. 8 for a description of the launch antenna). The FFT spectrum was obtained $201 \mathrm{~ms}$ into a $2 \mathrm{~ms}$ on, $2 \mathrm{~ms}$ off gyrotron pulse triggered $2.45 \mathrm{~s}$ into the TEXTOR shot when $300 \mathrm{~kW}$ was launched from each of two ICRH antennas and with $0.5 \mathrm{MW} \mathrm{NBI}$ in the cocurrent torodial direction. The lower graph shows the spectrum for the next identical plasma shot 100473 except that the gyrotron beam was diverted to a beam dump. The $29 \mathrm{MHz}$ component present in both these spectra is direct pickup by the measurement electronics and verifies that the measurement conditions were the same for obtaining both spectra. The interpretation of these spectra suggests that the gyrotron was perturbed by feedback through the gyrotron transmission line by a plasma ICRH modulated reflection. The insert figures show that when the gyrotron spectrum was perturbed, lower frequency amplitude fluctuations also increased in the forward power signal from a pickoff horn near the gyrotron output. 

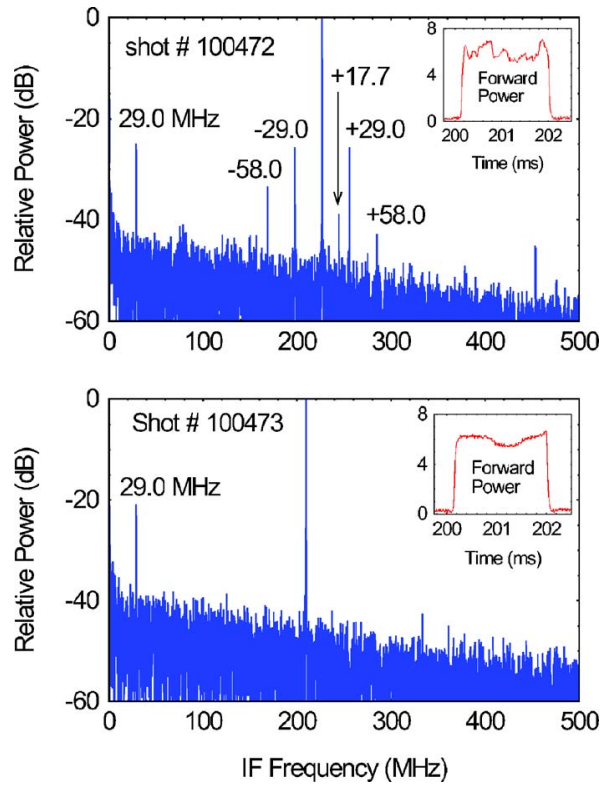

FIG. 4. Fast Fourier transform spectra of the heterodyne frequency downshifted TEXTOR $110 \mathrm{GHz}$ gyrotron. Top spectrum for plasma shot 100472 obtained with both ICRH antennas $(2 \times 300 \mathrm{~kW})$ and NBI $(1 \mathrm{MW})$. Bottom spectrum for the next plasma shot 100473 with same conditions, but the gyrotron beam diverted to a beam dump. Inserts show forward power signal. Spectra were obtained at $201 \mathrm{~ms}$ and the peak gyrotron frequency was measured to be $109.951 \mathrm{GHz}$ for these conditions on a previous shot.

In these initial measurements the gyrotron was not observed to be perturbed by the ICRH under different auxiliary heating combinations. With the gyrotron in the same $-5^{\circ}$ launch direction there was no perturbation when both ICRH antennas were on without NBI even when the ICRH power was higher at $2 \times 500 \mathrm{~kW}$, or when only one ICRH antenna was on with NBI. In another measurement the gyrotron launch direction was changed to $-30^{\circ}$ and no perturbation was observed with when both ICRH antennas where on with 1.2 MW NBI in the countercurrent direction. The polarization of the gyrotron was also adjusted during some of the measurements to optimize coupling to the characteristic propagation mode, which may not have been always achieved. More study will be needed to pinpoint the conditions and mechanism that cause this feedback, which seems be due to a driven plasma fluctuation under a very specific combination of auxiliary heating and gyrotron launch parameters.

\section{MEASUREMENTS AT ASDEX-UPGRADE}

The $105 \mathrm{GHz}$ mode of the Odissey-1 gyrotron was found to start at about $0.4 \mathrm{~ms}$ after the gyrotron trigger at a frequency of $104.786 \mathrm{GHz}$. For approximately $500 \mathrm{~kW}$ output power and a $2 \mathrm{~s}$ pulse, it chirped downward $104 \mathrm{MHz}$, as plotted in Fig. 5, on a logarithmic time scale. Most of the frequency downshift occurs in the first $100 \mathrm{~ms}$. At lower output power the frequency downshift has the same behavior, but the magnitude of the total downshift is reduced as indicated. For $335 \mathrm{~kW}$ the downshift was about $80 \mathrm{MHz}$ and for $180 \mathrm{~kW}$ it was about $60 \mathrm{MHz}$.

When the gyrotron was modulated at $20 \mathrm{~ms}$ on and $20 \mathrm{~ms}$ off the frequency varied, as shown in Fig. 6, for

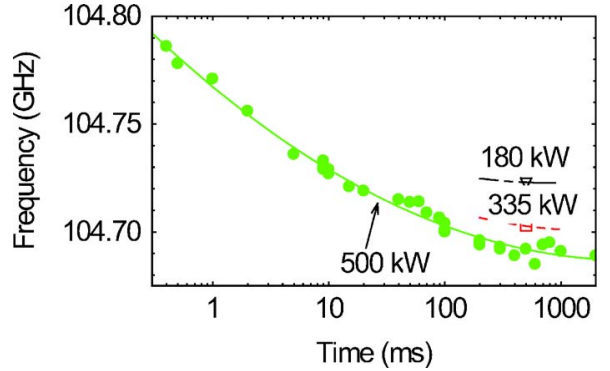

FIG. 5. Frequency dependence on pulse length and power of the $105 \mathrm{GHz}$ mode of the ASDEX Upgrade Odissey-1 gyrotron.

$500 \mathrm{~kW}$ peak power. The overall frequency downshift is the same as in the continuous pulse case except that it substantially recovers for each $20 \mathrm{~ms}$ pulse. This plot was obtained by a free running spectrum analyzer triggered with the gyrotron so the start instant of each $20 \mathrm{~ms}$ pulse was not always captured causing the ragged display of the peak frequency. The quick frequency recovery is due in part to millimeter wave turn on before the beam voltage stabilizes at the flat top and to efficient gyrotron cavity cooling. The importance of the latter effect was observed when the off time between pulses was reduced to less than $5 \mathrm{~ms}$ and the frequency recovery was seen to be much less.

The AUG gyrotron spectrum was also found to be relatively clean in the vicinity of the main peak and looked similar to the TEXTOR spectrum shown in Fig. 3 except without the spurious peaks. The observed linewidth of both the AUG and TEXTOR gyrotrons was found to be $120 \mathrm{kHz}$ full width at half maximum (FWHM), corresponding to the instrumental measurement limit.

\section{DISCUSSION}

The frequency characteristics of gyrotrons are important to understand when applying them to CTS diagnostics. In the case of the TEXTOR $110 \mathrm{GHz}$ gyrotron, the observed frequency downshift of about $20 \mathrm{MHz}$ during a pulse and spurious spectral components of about $\pm 17.5 \mathrm{MHz}$ around the main peak are small relative to the minimum receiver channel bandwidth of $80 \mathrm{MHz}$ and the notch filter bandwidth of about $300 \mathrm{MHz}$. These spectral characteristics of the $110 \mathrm{GHz}$ gyrotron are therefore not a significant issue for fast ion or for bulk ion CTS diagnostics, but would need to be taken into account for plasma rotation measurements be-

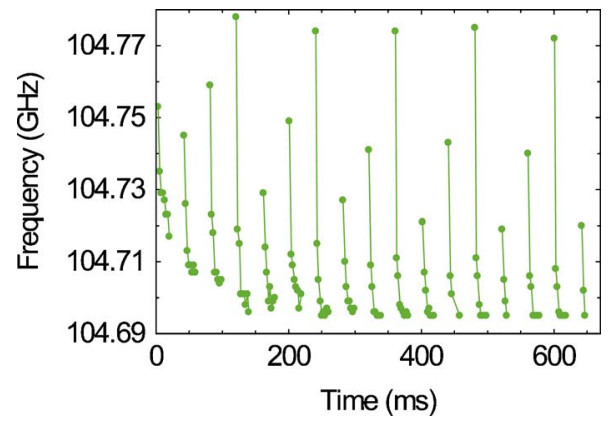

FIG. 6. Frequency dependence of the $105 \mathrm{GHz}$ Odissey-1 gyrotron when modulated $20 \mathrm{~ms}$ on and $20 \mathrm{~ms}$ off at $500 \mathrm{~kW}$. 
cause the maximum Doppler shift due to plasma rotation would be of the same order as the gyrotron frequency chirp. Of more concern for ion diagnostics is the observation of the spectral broadening by the ICRH frequency and its harmonics. The observed second harmonic peaks of $\pm 58 \mathrm{MHz}$ around the main peak combined with the frequency downshift make a much more challenging background spectrum to notch out. Also there may be even higher harmonics present beyond the $50 \mathrm{~dB}$ dynamic range of the present measurements but above the thermal CTS cross section of less than $-100 \mathrm{~dB}$. The observation that the gyrotron can be perturbed through a long optical transmission line by combined ICRH and NBI heating in a tokamak of itself is a phenomenon that warrants future study.

At AUG the observed larger frequency downshifts of the gyrotron of up to $100 \mathrm{MHz}$ are the same as the minimum $100 \mathrm{MHz}$ CTS receiver channel bandwidths and could be an issue for CTS diagnostics. However, the largest part of the frequency shift occurs in the first $0-0.5 \mathrm{~ms}$ of each pulse depending on the off time before (i.e., amount of cooling time of the cavity) and the voltage rise after turn on. This early portion of large frequency drift can be blocked by a millimeter wave switch in the receiver front end to limit signal acquisition to the main part of each pulse where the frequency is not changing significantly, typically less than $20 \mathrm{MHz}$. Therefore, with its narrow clean spectrum of over $500 \mathrm{~kW}$, the AUG two step gyrotron should be a good source for fast ion CTS diagnostics.

\section{ACKNOWLEDGMENTS}

This work was supported by U.S. DOE and EURATOM.

${ }^{1}$ S. B. Korsholm et al., Rev. Sci. Instrum., these proceedings.

${ }^{2}$ H. Bindslev, L. Porte, A. Hoekzema, J. Machuzak, P. Woskov, D. Van Ester, J. Egedal, and T. Hughes, Fusion Eng. Des. 53, 105 (2001).

${ }^{3}$ K. E. Kreischer, B. G. Danly, P. Woskoboinikow, W. J. Mulligan, and R. J. Temkin, Int. J. Electron. 57, 851 (1984).

${ }^{4}$ G. M. Batanov, L. V. Kolik, Yu. V. Novozhilova, M. L. Petelin, A. E. Petrov, K. A. Sarksyan, N. N. Skvortsova, and N. K. Kharchev, Tech. Phys. 46, 595 (2001).

${ }^{5}$ C. W. Peters et al., Phys. Rev. E 58, 6880 (1998).

${ }^{6}$ T. Idehara, S. Misudo, S. Satito, I. Ogawa, and S. Okajima, Rev. Sci. Instrum. 74, 2860 (2003).

${ }^{7}$ H. O. Prinz, Proceedings of the EURCON-2005-The International Conference on Computer as a Tool, 2005, Vol. 2, p. 1871.

${ }^{8}$ E. Westerhof et al., Fusion Sci. Technol. 47, 108 (2005).

${ }^{9}$ D. Wagner et al., The Joint 30th Conference on Infrared and Millimeter Waves, 2005, IEEE Catalog No. 05EX1150C, p. 24. 